\title{
The effect of turbulence strength on meandering field lines and Solar Energetic Particle event extents
}

\author{
Timo Laitinen ${ }^{1, *}$, Frederic Effenberger ${ }^{2,3}$, Andreas Kopp ${ }^{4}$ and Silvia Dalla ${ }^{1}$ \\ 1 Jeremiah Horrocks Institute, University of Central Lancashire, Preston, UK \\ ${ }^{2}$ International Space Science Institute, Bern, Switzerland \\ 3 Bay Area Environmental Research Institute, Petaluma, CA, USA \\ ${ }^{4}$ Université Libre de Bruxelles, Service de Physique Statistique et des Plasmas, CP 231, 1050 Brussels, Belgium
}

Received 23 May 2017 / Accepted 7 January 2018

\begin{abstract}
Insights into the processes of Solar Energetic Particle (SEP) propagation are essential for understanding how solar eruptions affect the radiation environment of near-Earth space. SEP propagation is influenced by turbulent magnetic fields in the solar wind, resulting in stochastic transport of the particles from their acceleration site to Earth. While the conventional approach for SEP modelling focuses mainly on the transport of particles along the mean Parker spiral magnetic field, multi-spacecraft observations suggest that the cross-field propagation shapes the SEP fluxes at Earth strongly. However, adding cross-field transport of SEPs as spatial diffusion has been shown to be insufficient in modelling the SEP events without use of unrealistically large cross-field diffusion coefficients. Recently, Laitinen et al. [ApJL 773 (2013b); $A \& A 591$ (2016)] demonstrated that the early-time propagation of energetic particles across the mean field direction in turbulent fields is not diffusive, with the particles propagating along meandering field lines. This early-time transport mode results in fast access of the particles across the mean field direction, in agreement with the SEP observations. In this work, we study the propagation of SEPs within the new transport paradigm, and demonstrate the significance of turbulence strength on the evolution of the SEP radiation environment near Earth. We calculate the transport parameters consistently using a turbulence transport model, parametrised by the SEP parallel scattering mean free path at $1 \mathrm{AU}, \lambda_{\|}^{*}$, and show that the parallel and cross-field transport are connected, with conditions resulting in slow parallel transport corresponding to wider events. We find a scaling $\sigma_{\varphi, \max } \propto\left(1 / \lambda_{\|}^{*}\right)^{1 / 4}$ for the Gaussian fitting of the longitudinal distribution of maximum intensities. The longitudes with highest intensities are shifted towards the west for strong scattering conditions. Our results emphasise the importance of understanding both the SEP transport and the interplanetary turbulence conditions for modelling and predicting the SEP radiation environment at Earth.
\end{abstract}

Keywords: Cosmic rays / diffusion / Sun: heliosphere / Sun: particle emission / turbulence

\section{Introduction}

Solar Energetic Particles (SEPs), accelerated in solar eruptive events, pose a significant space weather threat to manmade technology and astronauts (Committee on the Evaluation of Radiation Shielding for Space Exploration, 2008). To forecast SEP fluxes near Earth's orbit, it is important to understand how their acceleration is related to flares, coronal mass ejections and other related phenomena during solar eruptions. Furthermore, as the particles propagate through a turbulent solar wind medium, predicting the fluxes and fluences at $1 \mathrm{AU}$ requires understanding of how the solar wind turbulence affects the charged particle motion.

\footnotetext{
*Corresponding author: tlmlaitinen@uclan.ac.uk
}

The propagation of SEPs in a turbulent medium is typically modelled as random walk due to the stochastic nature of magnetic field fluctuations, and described as spatial and velocity diffusion using a Fokker-Planck formalism (Parker, 1965; Jokipii, 1966). The propagation along the mean field is usually modelled as either spatial or pitch angle diffusion (Jokipii, 1966). The cross-field transport, on the other hand, is usually described as spatial diffusion due to random walk of the turbulent magnetic field lines (Jokipii, 1966), compounded by the parallel scattering (Matthaeus et al., 2003; Shalchi, 2010; Ruffolo et al., 2012). These approaches have support in full-orbit particle simulations (Giacalone and Jokipii, 1999) and galactic cosmic ray observations (Burger et al., 2000; Potgieter et al., 2014). However, several recent observational studies suggest faster propagation of SEPs across the mean field than predicted by the current theoretical understanding: 
they often require a ratio of the cross-field diffusion coefficient to the parallel one of order $\kappa_{\perp} / \kappa_{\|} \sim 0.1-1$ (Zhang et al., 2003; Dresing et al., 2012; Dröge et al., 2014) ${ }^{1}$, whereas values $\kappa_{\perp} / \kappa_{\|} \lesssim 0.01$ are more consistent with the interplanetary turbulence conditions at 1 AU (Burlaga and Turner, 1976; Pei et al., 2010; Laitinen et al., 2016).

Recently, Laitinen et al. (2013b, L2013 in the following) demonstrated, using full-orbit particle simulations in turbulent magnetic fields superposed on a constant background magnetic field, that SEPs can propagate rapidly to large cross-field distances along turbulently meandering field-lines already early in SEP event history. While the concept of field-line meandering is included in earlier models in the diffusion coefficient, L2013 showed that the initial SEP cross-field transport is non-diffusive, and cannot be modelled using a diffusion approach. As further shown in Laitinen and Dalla (2017), the particles remain on their initial meandering field lines up to tens of hours before decoupling and spreading more freely across the meandering field lines. Thus, the initial evolution of SEP events is dominated by systematic widening of the SEP cross-field distribution, while diffusion dominates the evolution of the SEP cross-field distribution only tens of hours after the SEP injection. L2013 pointed out also that the early-time non-diffusive SEP propagation across the mean field direction is much faster than the time-asymptotic crossfield diffusion.

Using the novel modelling approach introduced in L2013, Laitinen et al. (2016) developed a particle transport model in the heliospheric Parker Spiral magnetic field configuration. They demonstrated that in moderate turbulence conditions, parametrised by the parallel scattering mean free path $\lambda_{\|}=0.3 \mathrm{AU}$, fast spreading of SEPs across the field to a wide range of longitudes, as found by multi-spacecraft observations of SEP events (e.g. Lario et al., 2006; Dresing et al., 2012; Dresing et al., 2014; Richardson et al., 2014), could be obtained already with a narrow source region.

Laitinen et al. (2016) considered a case study of SEP propagation in magnetic turbulence characterised by the value of the parallel scattering mean free path at $1 \mathrm{AU}$, $\lambda_{\|}^{*} \equiv \lambda_{\|}(r=1 \mathrm{AU})=0.3 \mathrm{AU}$, whereas in reality the turbulence, and as a consequence the particle transport parameters, can vary considerably from event to event (Burlaga and Turner, 1976; Bavassano et al., 1982; Palmer, 1982; Wanner and Wibberenz, 1993), and even be different during an event at different heliographic longitudes (Dröge et al., 2016). Using full-orbit simulations with a constant background magnetic field, Laitinen and Dalla (2017) and Laitinen et al. (2017) showed that the initial cross-field extent of the SEP distribution depends strongly on the turbulence amplitude. Thus, it is important to evaluate the effect of different levels of turbulence amplitudes on the SEP event width in the Parker Spiral geometry. In this study, we compare longitudinal SEP event extents for different levels of plasma turbulence, as parametrised by parallel mean free paths $\lambda_{\|}^{*}$. We concentrate on $10 \mathrm{MeV}$ protons, which have received less attention in multispacecraft-observed SEP event modelling. While electrons and protons are often considered to be accelerated in different

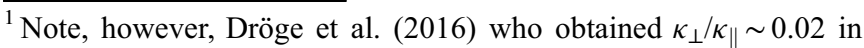
some cases.
}

processes and source regions (e.g. Reames, 1999), the recently observed similar heliolongitudinal extents for electrons and protons during different events (e.g. Richardson et al., 2014) may suggest similar processes responsible for the spreading of these particles in interplanetary space, warranting closer analysis of their cross-field transport. We present the employed models in Section 2, the results in Section 3 and discuss and draw our conclusions in Sections 4 and 5.

\section{Models}

The Fokker-Planck and Field Line Random Walk (FP + FLRW) model used in this study is based on the findings of L2013, who used full-orbit particle simulations in turbulent magnetic fields to show that the initial cross-field propagation of charged particles in turbulent magnetic fields is nondiffusive. The particles tend to follow their field lines, which spread across the mean field direction due to turbulent fluctuations. Until a particle decouples from its field line, its propagation across the mean background magnetic field is deterministic, in the sense that particles which scatter in their pitch angle from one pitch angle hemisphere to the other will just retract their path along the same stochastically meandering path. Thus, the particle cross-field transport behaviour remains non-Markovian at times shorter than the timescale over which the particle decouples from its original field line. L2013, and subsequently Laitinen and Dalla (2017) and Laitinen et al. (2017) showed that this non-Markovian propagation can dominate an SEP event for up to tens of hours, depending on the turbulence conditions. The slow onset of the decoupling of particles from the meandering field lines can explain the intensity dropouts observed in some SEP events (e.g. Mazur et al., 2000), as shown for example in simulations by Tooprakai et al. (2016).

While the motion resulting from field-line meandering and field-parallel scattering has been described as compound (sub) diffusion by earlier researchers (e.g. Kóta and Jokipii, 2000), L2013 discovered that during early times, the particle propagation cannot be described as diffusion at all, as the particles retain memory of their propagation history.

L2013 introduced the FP + FLRW model, where this process is described as combination of particle propagation along meandering path (supplemented with pitch angle scattering) and particle diffusion across the meandering field. As shown in L2013 and subsequently further investigated in Laitinen et al. (2017), the model agrees well with full-orbit simulations at early times, when the particles are still tied to their field lines, and at the time-asymptotic limit, where the particle cross-field propagation is fully diffusive.

In the full-orbit simulations in L2013, the meandering of field lines was created by using a superposition of Fourier modes corresponding to spectra of slab- and 2D-mode waves, which can be cumbersome particularly in scenarios more complex than the constant background field used in that work. For simpler and faster Fokker-Planck description of particle propagation, the FP + FLRW mode considers a description of diffusively meandering particle paths, based on results of, e.g., Matthaeus et al. (1995). In the FP + FLRW approach, rather than calculating the complete fluctuating magnetic field, the effect of diffusive spreading field lines on the propagating 
particles is estimated by propagating each particle on a separately drawn stochastic meandering path. Thus, schematically the algorithm of the FP + FLRW model for each pseudoparticle in the simulations proceeds as follows:

1. calculate a diffusively meandering path, unique to the particle being simulated;

2. propagate a pseudo-particle until end of simulation time using the following scheme:

(a) step along the meandering path;

(b) diffuse and adiabatically focus the pitch angle. Here, for the focusing the mean background magnetic field is used;

(c) take a diffusive cross-field spatial step in direction perpendicular to the meandering path.

It should be emphasised that in the FP + FLRW model each simulated pseudo-particle has only one meandering field line, and the particle diffuses across this meandering field line: The pseudo-particle does not switch from one meandering path to another. The individual particles propagating each at their unique meandering paths facilitate the initial non-diffusive evolution of the particle distribution seen in L2013 and Laitinen et al. (2017), whereas the particle's spatial cross-field diffusion from this meandering path facilitates the timeasymptotic diffusive particle transport.

An alternative approach, with a particle changing from one meandering path to another, could also be devised. However, such a model would require precise description of the decoupling of particles from their initial fieldlines, and the relation of that decoupling process to the turbulent field line separation, which are not yet well understood. Thus, as the simpler FP + FLRW model was shown by L2013 to reproduce the full-orbit particle simulations well, the single-meanderingpath approach is well-justified.

The stochastically meandering path is described as fieldline diffusion using a stochastic differential equation (SDE, Gardiner, 2009; Strauss and Effenberger, 2017), with the displacement $d r_{\perp}$ across the Parker field direction given as

$$
d r_{\perp}=\sqrt{2 D_{\mathrm{FL}}(r) d r_{\|}} W_{\perp}
$$

where $d r_{\|}$is a step along the local Parker spiral direction, and $W_{\perp}$ a Gaussian random number with zero mean and unit variance. The field-line diffusion coefficient $D_{\mathrm{FL}}(r)$ is calculated based on Matthaeus et al. (1995), using the radially-evolving 2D component of the turbulence spectrum discussed below.

This method of calculating the meandering field line using a stochastic method is naturally statistic in nature, and does not reproduce patchy spatial particle distributions seen in some full-orbit particle simulations (e.g. Tooprakai et al., 2016), which may explain intensity dropouts observed in SEPs (e.g. Mazur et al., 2000) (see also discussion in Laitinen and Dalla, 2017). It also cannot replicate the coherence of nearby field lines, but, as shown in (Ruffolo et al., 2004), such coherence is lost in 2D-dominated turbulence at scales which are small compared to heliospheric scales.

The particle propagation along meandering field lines, the step B in the FP + FLRW scheme, is performed using an SDE formulation of the Fokker-Planck equation (Roelof, 1969; Skilling, 1971; Isenberg, 1997; Zhang et al., 2009;
Strauss and Effenberger, 2017). The Fokker-Planck equation is given as

$$
\begin{aligned}
\frac{\partial f}{\partial t} & +\left(\mu \nu \mathbf{b}_{m}+\mathbf{V}_{s w}\right) \cdot \nabla f+\frac{v}{2 L}\left(1-\mu^{2}\right) \frac{\partial f}{\partial \mu} \\
& +\left[\frac{\mu\left(1-\mu^{2}\right)}{2}\left(\nabla \cdot \mathbf{V}_{s w}-3 \mathbf{b b}: \nabla \mathbf{V}_{s w}\right)\right] \frac{\partial f}{\partial \mu} \\
& +\left[\frac{1-3 \mu^{2}}{2} \mathbf{b b}: \nabla \mathbf{V}_{s w}-\frac{1-\mu^{2}}{2} \nabla \cdot \mathbf{V}_{s w}\right] p \frac{\partial f}{\partial p} \\
& =\frac{\partial}{\partial \mu}\left(D_{\mu \mu} \frac{\partial f}{\partial \mu}\right)+\nabla \cdot \hat{\kappa} \nabla f+Q(r, v, t),
\end{aligned}
$$

where $v$ and $\mu$ are the particle's velocity and pitch-angle cosine, respectively, and $Q$ is the particle source function. $\mathbf{V}_{\mathrm{sw}}$ is the solar wind velocity, $\mathbf{b}$ a unit vector along the Parker magnetic field $\mathbf{B}$, and $\mathbf{b}_{m}$ a unit vector along the meandering path given by equation (1). The focusing length $L=-B /(\partial B /$ $\partial s$ ), with $s$ the arc-length along the field-line, is calculated using the mean Parker spiral field. The particles scatter in pitch angle cosine $\mu=v_{\|} / v$ according to the pitch-angle diffusion coefficient $D_{\mu \mu}$, and across the mean field direction according to the spatial cross-field diffusion coefficient $\kappa_{x x}=\kappa_{y y} \equiv \kappa_{\perp}$, the non-zero elements of the cartesian diffusion tensor $\hat{\kappa}$. In this study, we ignore the energy changes given by the 5 th term in equation (2), as they are expected to be small during the early phase of the SEP event (e.g. Dalla et al., 2015). The remaining equation is solved using the SDE code described in further detail in Kopp et al. (2012).

The particles are propagated along a path that consists of a Parker spiral field superposed with stochastic fluctuations, resulting in particle paths that meander about the Parker spiral. The magnitude of the magnetic field is taken as the mean Parker spiral field value,

$$
B(r)=B_{0}\left(\frac{r_{0}}{r}\right)^{2} \sqrt{\frac{r^{2}+a^{2}}{r_{0}^{2}+a^{2}}},
$$

where $B_{0}=5 \mathrm{nT}$ is the magnetic field strength at $r_{0}=1 \mathrm{AU}$, and $a=V_{s w} /\left(\Omega_{\odot} \quad\right.$ sin $\left.\theta\right)$, where $V_{s w}=400 \mathrm{~km} / \mathrm{s}, \Omega_{\odot}=$ $2.8631 \cdot 10^{-6} \mathrm{rad} / \mathrm{s}$ is the solar rotation rate and $\theta$ the co-latitude.

As shown by L2013 and Laitinen and Dalla (2017), at intermediate timescales, of the order of the parallel scattering timescale of the particles, the particles begin to decouple from their field lines and eventually time-asymptotically approach diffusive cross-field propagation. We include the transition to the time-asymptotic cross-field diffusion into our simulations by diffusing the particles across the meandering field. While this approach is not precise, L2013 demonstrated that it is much more accurate than using only cross-field diffusion from the mean field, or only particle propagation along meandering field lines. The cross-field diffusion coefficient $\kappa_{\perp}$ is calculated using the Non-Linear Guiding Centre theory (NLGC, Matthaeus et al., 2003) for the spectrum described below. We do not incorporate the recently suggested dependence of $\kappa_{\perp}$ on the particle's pitch angle (Dröge et al., 2010; Qin and Shalchi, 2014; Strauss and Fichtner, 2015) since full-orbit results (Laitinen and Dalla, 2017) indicate that it might be more complicated than the suggested proportionality to $|\mu|$ or $1-\mu^{2}$.

It should be noted that although both the meandering path of the particle and the cross-field diffusion coefficient are 

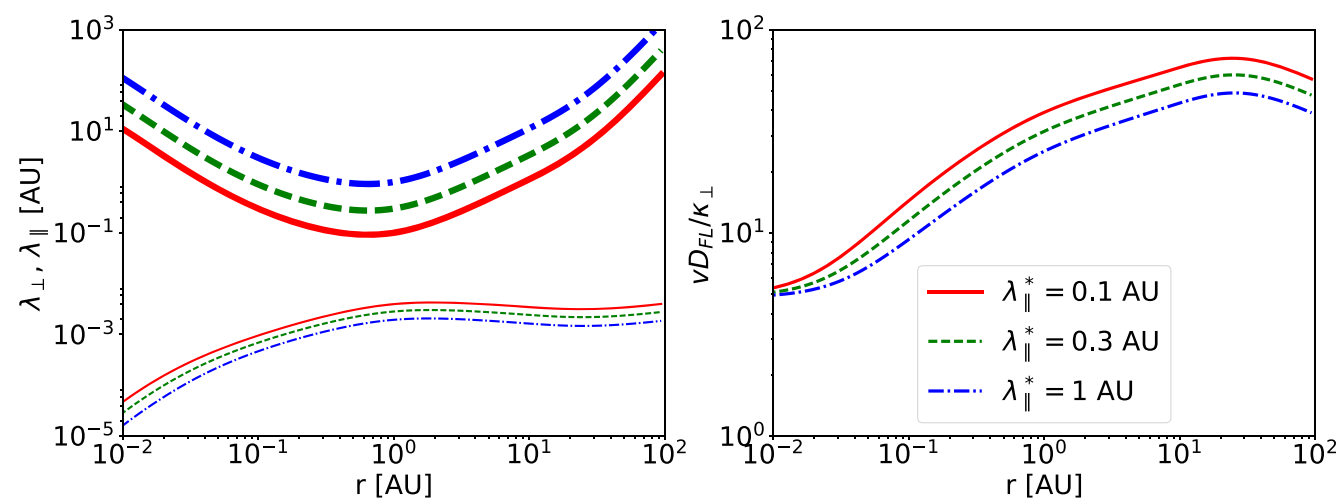

Fig. 1. Left panel: Parallel (thick curves) and perpendicular (thin curves) particle mean free paths as function of radial distance from the Sun, for $10 \mathrm{MeV}$ protons and different turbulence strengths parametrised by $\lambda_{\|}^{*}$. Right panel: The ratio of the field line and cross-field particle diffusion coefficients.

calculated from the same turbulence spectrum, this does not amount to taking the effect of meandering field lines on particles into account twice. As discussed in Laitinen and Dalla (2017), the initial cross-field spreading of the particles is caused by the particles following the meandering field lines. The cross-field diffusion, on the other hand is caused by particles decoupling from the field lines and following new field lines, which meander relative to the original field line (see also Ruffolo et al., 2012). Thus, the two phenomena, while both related to field-line meandering, are separate and must be both accounted for.

Finally, the particles also scatter as they propagate along the meandering field line. We model this by using a quasilinear pitch-angle diffusion coefficient $D_{\mu \mu}$ (e.g. Jokipii, 1966), with additional scattering at $\mu=0$ to close the resonance gap, as suggested by Beeck and Wibberenz (1986) (see Laitinen et al. (2016) for details).

The particle and field line diffusion coefficients are calculated using a heliospheric turbulence spectrum with slab and 2D components (Gray et al., 1996). The turbulence spectrum is given as

$$
S(\mathbf{k}) \equiv S\left(\mathbf{k}, r_{0}\right)=S_{\perp}\left(k_{\perp}, r_{0}\right) \delta\left(k_{\|}\right)+S_{\|}\left(k_{\|}, r_{0}\right) \delta\left(\mathbf{k}_{\perp}\right),
$$

where $k_{\|}$and $k_{\perp}=\left|\mathbf{k}_{\perp}\right|$ are the parallel and perpendicular wavenumbers, and $S_{\|}\left(k_{\|}\right)$and $S_{\perp}\left(k_{\perp}\right)$ are broken power law spectra as given in Laitinen et al. (2016). It should be noted that our turbulence model differs from the one introduced by Giacalone (2001), in which the turbulence is generated by motion of magnetic field footpoints due to solar supergranulation. The latter does not allow for further turbulence evolution of the magnetic fields in interplanetary space (e.g. Bruno and Carbone, 2013, and references therein), and thus limits the meandering of interplanetary field lines to the angular scale of the supergranular motion.

We model the radial evolution of the turbulence within the heliosphere using the WKB transport approximation (Richter and Olbers, 1974; Tu et al., 1984). For simplicity, we neglect wave refraction, changes in the wave geometry and the modulus of $k$, as well as non-linear evolution of the spectral shape (see Laitinen et al., 2016, for discussion). We further consider constant radial solar wind velocity $V_{s w, r 0}$, and electron density $n_{e}(r)=n_{e 0} r_{0}^{2} / r^{2}$. With these assumptions, the radial evolution of the turbulence spectrum can be written as

$$
S_{\|, \perp}\left(k_{\|, \perp}, r\right)=S_{\|, \perp}\left(k_{\|, \perp}, r_{0}\right)\left(\frac{r_{0}}{r}\right)^{3}\left(\frac{V_{s w, r 0}+v_{A 0}}{V_{s w, r 0}+\frac{r_{0}}{r} v_{A 0}}\right)^{2},
$$

where $V_{s w, r 0}=400 \mathrm{~km} / \mathrm{s}$ is the constant solar wind velocity, and subscript 0 denotes the values at reference distance $r_{0}$, and $v_{a}$, $r_{0}=30 \mathrm{~km} / \mathrm{s}$ is the Alfvén velocity at $r_{0}=1 \mathrm{AU}$. The resulting $\propto r^{3}$ trend of the wave power is consistent with turbulence observations (e.g. Bavassano et al., 1982).

The spectral power of the slab and 2D components, $S_{\|, \perp}\left(k_{\|, \perp}, r_{0}\right)$, is parametrised by the total turbulence amplitude $\delta B^{2}=2 \int S(\mathbf{k}) d \mathbf{k}$, and the energy ratio $\delta B_{\|}^{2} / \delta B_{\perp}^{2}$ between the slab and 2D modes, for which we use $20 \%: 80 \%$, as suggested by Bieber et al. (1996). The total turbulence amplitude is parametrised by the parallel mean free path at $1 \mathrm{AU}$, $\lambda_{\|}^{*} \equiv \lambda_{\|}(r=1 \mathrm{AU})$, as given by the quasilinear theory (Jokipii, 1966) for the slab spectrum $S_{\|}\left(k_{\|}\right)$at 1 AU. It should be emphasised that the parallel mean free path is fixed using $\lambda^{*}$ only at 1 AU: elsewhere all particle and field line transport parameters are calculated consistently using the turbulence model given by equations (4) and (5). Thus, we constrain the radial evolution of both parallel and perpendicular transport parameters consistently, instead of using an ad hoc parametrisation.

The parallel and cross-field mean free paths for $10 \mathrm{MeV}$ protons are shown as a function of distance from the Sun in the left panel of Figure 1 for the modelled turbulence corresponding to $\lambda_{\|}^{*}$ values of $0.1,0.3$ and $1 \mathrm{AU}$. Close to the Sun, the parallel mean free path is large, indicating nearly scatter-free propagation, and decreases to the parametrised value $\lambda_{\|}^{*}$ at $1 \mathrm{AU}$, after which it increases again. On the other hand, the cross-field mean free path is very short close to the Sun and increases initially faster than $\propto r$, indicating that the diffusion coefficient ratio $\kappa_{\perp} / \kappa_{\|}$is not constant in the heliosphere, but depends strongly on the radial distance from the Sun. Similar results of the radial dependence of the particle diffusion coefficients have recently been presented in several studies (Pei et al., 2010; Chhiber et al., 2017; Strauss et al., 2017).

In the right panel of Figure 1, we describe how the crossfield particle and field line diffusion coefficients evolve in the 


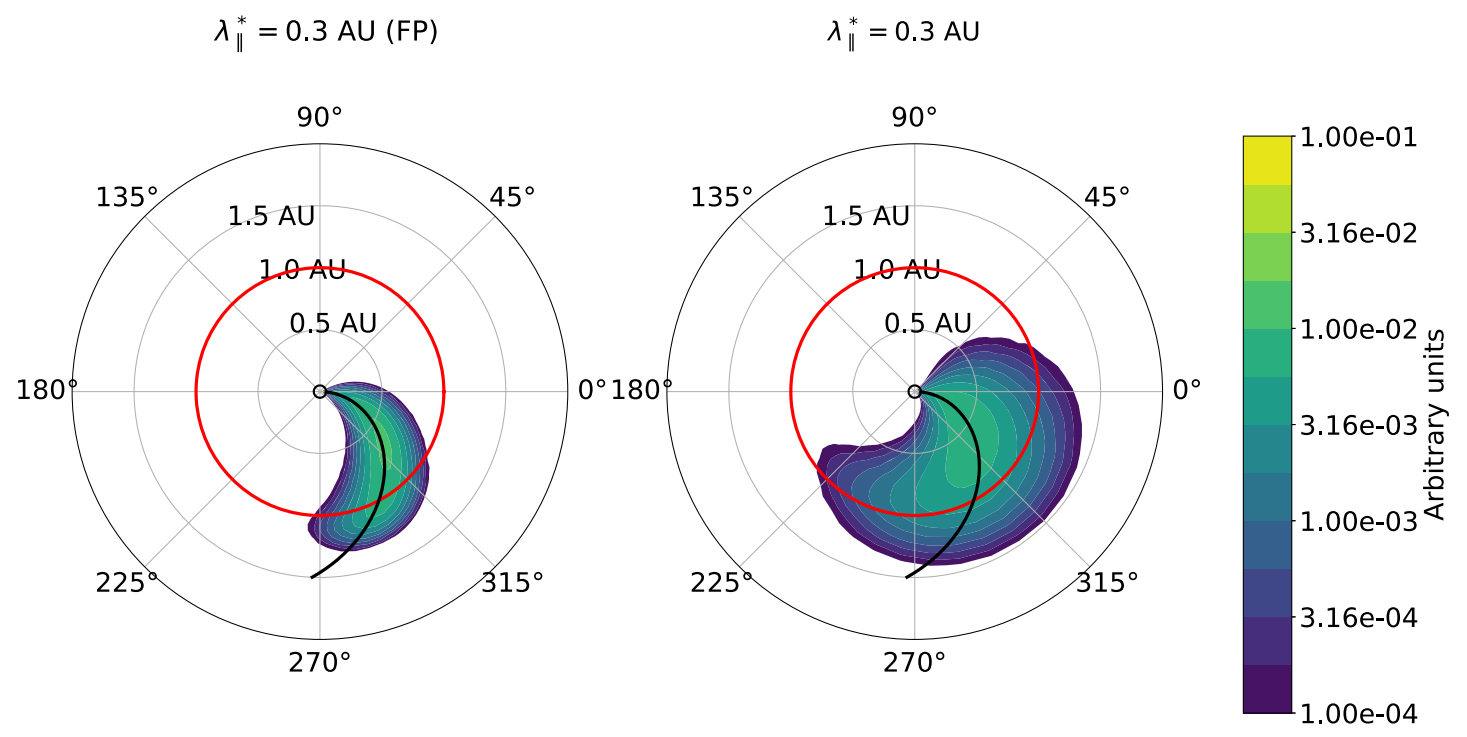

Fig. 2. Distribution of $10 \mathrm{MeV}$ SEPs integrated between latitudes $-10^{\circ}$ and $10^{\circ}$, in arbitrary units, $2 \mathrm{~h}$ after impulsive injection at $(r, \varphi, \theta)=\left(1 r_{\odot}\right.$, $0, \pi / 2$ ), for the FP model (left panel) and the FP + FLRW model (right panel), respectively, with $\lambda_{\|}^{*}=0.3$ AU. The red curve depicts 1 AU radial distance, and the thick black spiral curve the Parker field connected to the injection location.

heliosphere, by presenting their ratio as a function of radial distance from the Sun. As discussed in L2013, particles propagate initially along meandering field lines that spread diffusively according to diffusion coefficient $D_{\mathrm{FL}}$. Timeasymptotically, the cross-field propagation is diffusive, described by the particle cross-field diffusion coefficient $\kappa_{\perp}$, which is much slower than the spreading of particles nondiffusively along the field lines, due to particles scattering along the meandering field lines. As can be seen in the right panel of Figure 1, the spreading of particles across the field due to the early process, at rate $v D_{\mathrm{FL}}$, is 1-2 orders of magnitude faster than the time-asymptotic diffusive cross-field spreading of the particles, and the difference increases as a function of distance from the Sun. The ratio $v D_{\mathrm{FL}} / \kappa_{\perp}$ decreases for weaker turbulence (larger $\lambda_{\|}^{*}$ ), and, as discussed in Laitinen et al. (2016), $v D_{\mathrm{FL}}$ and $\kappa_{\perp}$ calculated using the NLGC (Matthaeus et al., 2003) converge to the same value in the limit of no parallel scattering for a particle beam.

\section{Results}

We study the effect of turbulence strength on SEP event evolution in time, both along and across the mean field direction. We use a simple injection profile

$$
\begin{aligned}
Q(r, \theta, \phi, t) & =\delta\left(r-1 \mathrm{r}_{\odot}\right) \delta(\theta-\pi / 2) \delta(\mu-1) \cdot \delta(E \\
& \left.-E_{0}\right) \delta(\phi) \delta(t),
\end{aligned}
$$

where $(r, \theta, \varphi)$ define the spherical coordinate system, $r_{\odot}$ is the solar radius, and $E_{0}=10 \mathrm{MeV}$ the energy of the simulated protons. The coronal magnetic field can be complicated, varying considerably from event to event. However, in this study we are interested in SEP propagation in general, instead of during a particular SEP event. For this reason, we model the coronal magnetic field simply as a Parker spiral starting from the injection height at $1 r_{\odot}$, reserving case studies that investigate the spatial structure of the source region of particles for future work.
The results of our study can be extended to other injection profiles by simply convolving the impulse response with more complicated injection profiles. It should be noted that Strauss et al. (2017) demonstrated recently that the source size at or near the Sun plays only a minor role in the cross-field extent of an SEP event in cases where cross-field propagation of particles is efficient. Thus, our results can be considered to represent the SEP event evolution as injected from a narrow to an intermediate-size SEP source region.

We first show an overview of the early SEP event extent in Figures 2 and 3, as a snapshot of the spatial distribution of $10 \mathrm{MeV}$ protons in the inner heliosphere, two hours after the injection. The red circle depicts Earth's orbit, and the black spiral curve the Parker spiral connected to the injection location. The SEP distribution is given as a function of heliolongitude and the heliocentric radial distance, integrated over latitudes $\pm 10^{\circ}$.

In Figure 2, we present the SEP distributions for turbulence parametrised with $\lambda_{\|}^{*}=0.3 \mathrm{AU}$. The left panel depicts the model where the field-line meandering is omitted (the FP model in Laitinen et al., 2016), whereas the right panel is obtained from the model described in Section 2. As discussed in Laitinen et al. (2016), the primary effect of including the field line meandering into the modelling is that the particles spread rapidly across the mean Parker field direction to a wide range of heliolongitudes, as compared to the slow spreading of the SEPs across the mean field depicted in the left panel of Figure 2.

In Figure 3, we show the effect of changing the turbulence strength on the radial and cross-field extent of an SEP event. In the left panel, the turbulence amplitude has been increased to result in stronger parallel scattering conditions, as parametrised by $\lambda_{\|}^{*}=0.1 \mathrm{AU}$. The differences with the $\lambda_{\|}^{*}=0.3$ AU case in the right panel of Figure 2 are notable. The strong parallel scattering of the SEPs prevents the particles from propagating as far into the heliosphere as in the $\lambda_{\|}^{*}=0.3 \mathrm{AU}$ case. On the other hand, the core of the SEP distribution in the 


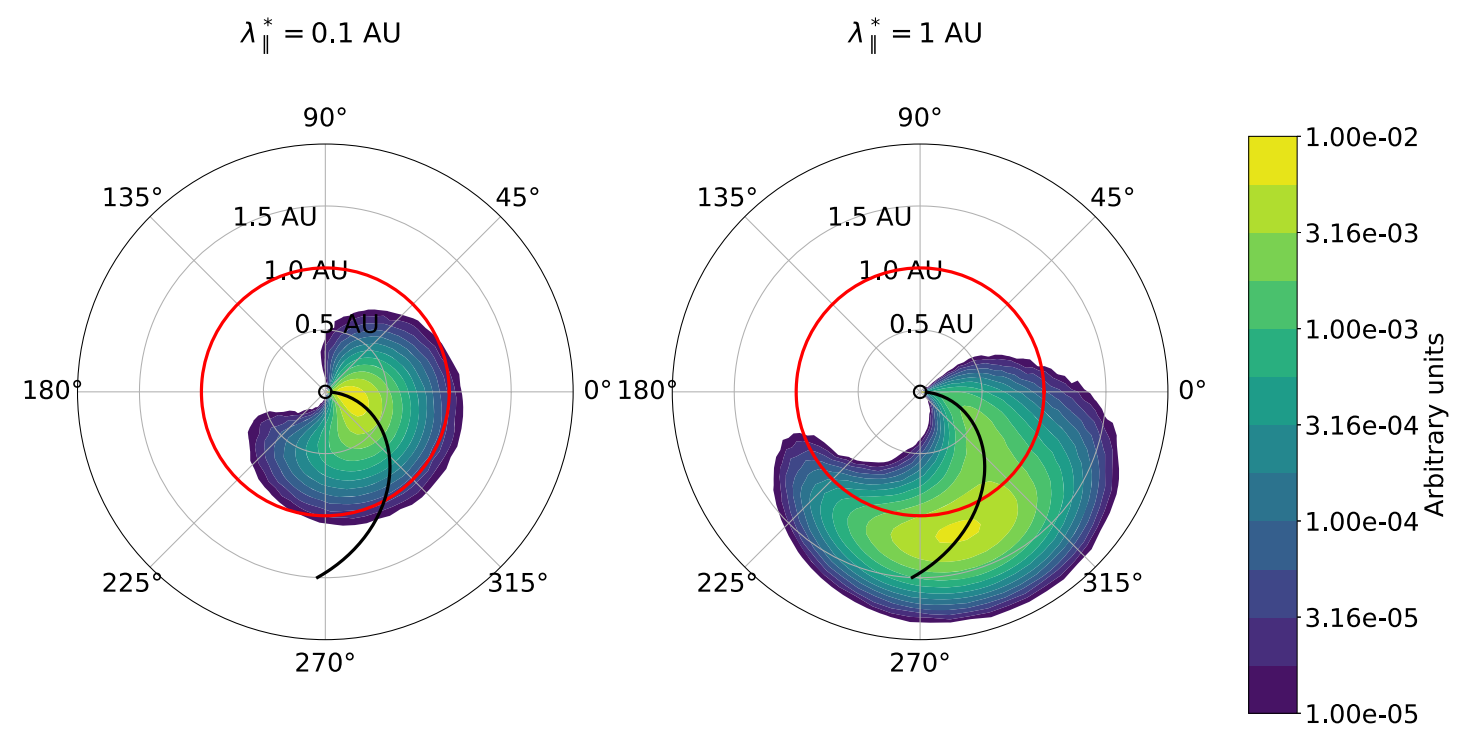

Fig. 3. Distribution of $10 \mathrm{MeV}$ SEPs integrated between latitudes $-10^{\circ}$ and $10^{\circ}$, as in Figure 2 , with left panel for FP $+\mathrm{FLRW}$ with $\lambda_{\|}^{*}=0.1 \mathrm{AU}$, and right panel for FP+FLRW with $\lambda_{\|}^{*}=1 \mathrm{AU}$.

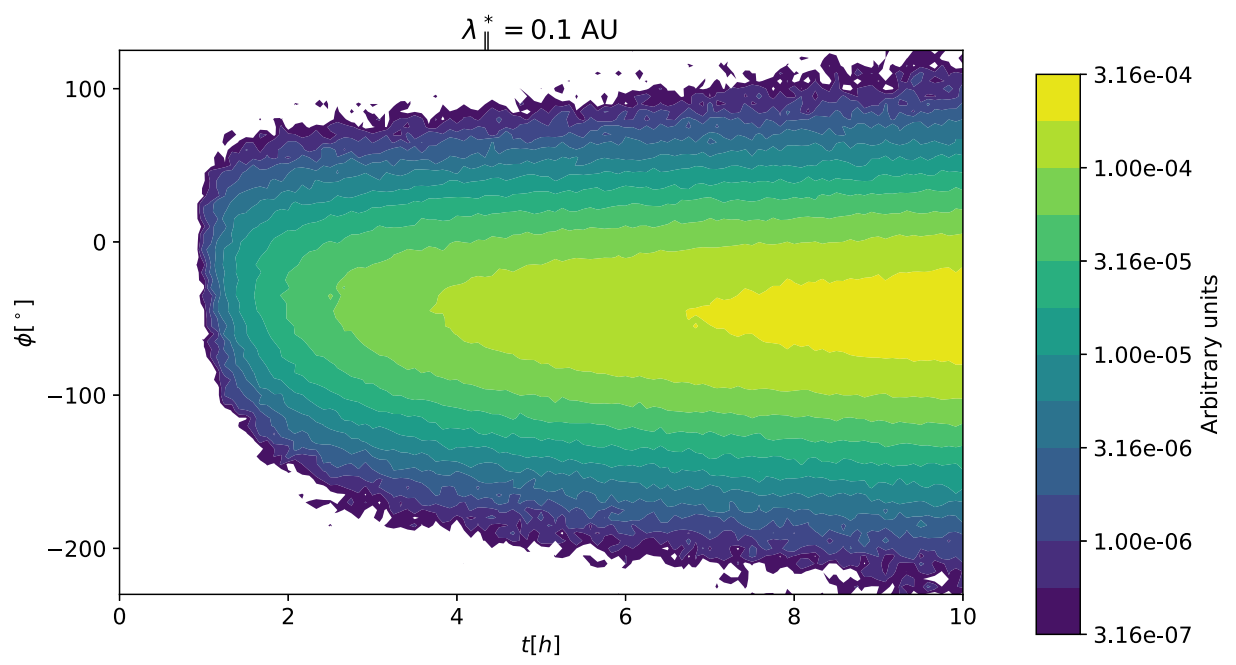

Fig. 4. $10 \mathrm{MeV}$ SEP intensity at $1 \mathrm{AU}$, as a function of time and heliographic longitude, with magnetic connection along the Parker spiral at $\varphi=-62^{\circ}$, for $\lambda_{\|}^{*}=0.1 \mathrm{AU}$.

left panel of Figure 3 is considerably wider. This is caused by the particles following the meandering field lines which diffuse with $D_{F L} \propto \delta B / B$ in 2D-dominated turbulence (Matthaeus et al., 1995). Thus, while particle propagation along the mean field line is inhibited by strong scattering in stronger turbulence, the cross-field transport of the SEP distribution is enhanced by the stronger meandering of the field lines.

For weaker turbulence (the $\lambda_{\|}^{*}=1$ AU case), presented in the right panel of Figure 3, we see that the reduced parallel scattering of the SEPs causes an increased radial extent of the particle distribution: the front of the SEP population has propagated to nearly $2 \mathrm{AU}$ from the Sun, consistent with nearly-scatter-free propagation of $10 \mathrm{MeV}$ protons of $\sim 1 \mathrm{AU} /$ $\mathrm{h}$. On the other hand, the population is narrower in the crossfield direction, as compared to the cases with $\lambda_{\|}^{*}=0.3 \mathrm{AU}$ and 0.1 AU. This is due to the reduced turbulent meandering of the field lines in weaker turbulence.
In Figures 4 and 5, we show the evolution of the SEP event at $1 \mathrm{AU}$ as a function of heliographic longitude and time, for $\lambda_{\|}^{*}=0.1 \mathrm{AU}$ and $1 \mathrm{AU}$, respectively. In both cases, the particles spread rapidly across the mean field direction, with the onset seen at a wide range of longitudes within the first $2 \mathrm{~h}$ of the event. In the strong scattering case (Fig. 4), the diffusive nature of the cross-field propagation of the particles after the initial fast spreading results in the parabole shape of the highintensity contours as a function of time and longitude, which suggest the diffusive scaling of the longitudinal variance of the particles as $\sigma_{\phi}^{2}(t) \propto t$ after the initial fast expansion along the meandering field lines.

The low scattering case (Fig. 5) is very different from the stronger scattering case shown in Figure 4. The proton intensity increases rapidly to its maximum value during the first two hours from the injection, and then begins to decay. This is due to the particles being nearly scatter-free and 


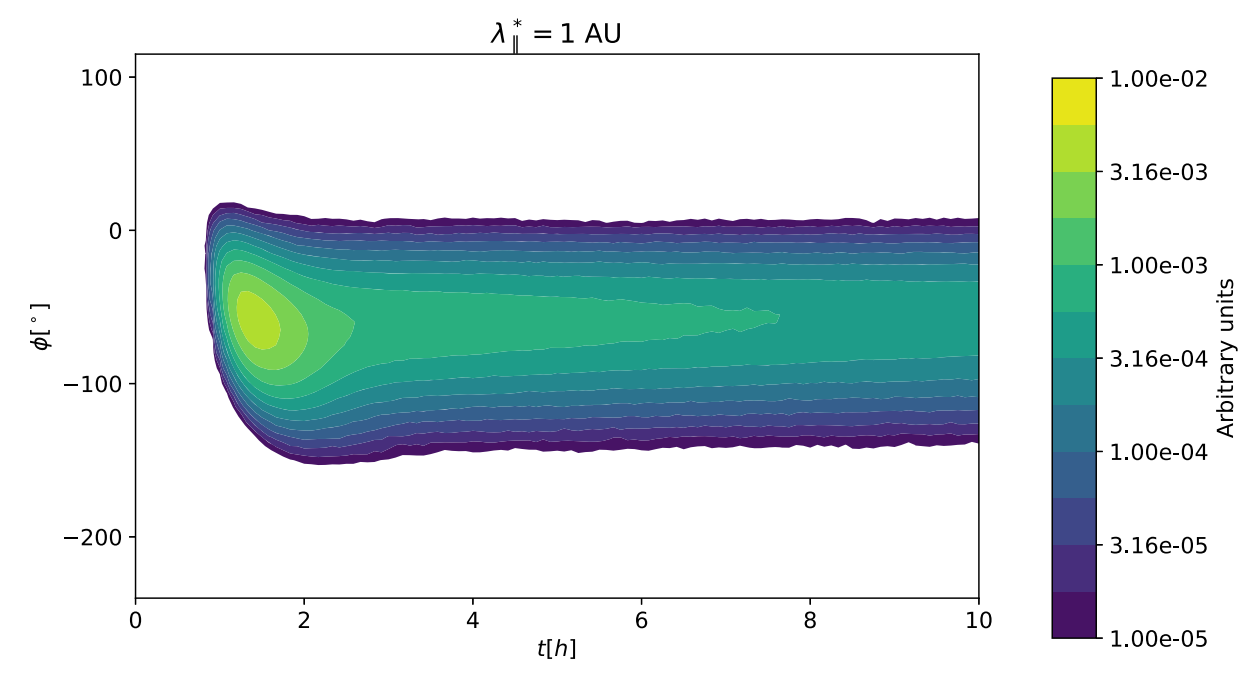

Fig. 5. $10 \mathrm{MeV}$ SEP intensity at $1 \mathrm{AU}$, as a function of time and heliographic longitude, with magnetic connection along the Parker spiral at $\varphi=-62^{\circ}$, for $\lambda_{\|}^{*}=1 \mathrm{AU}$.

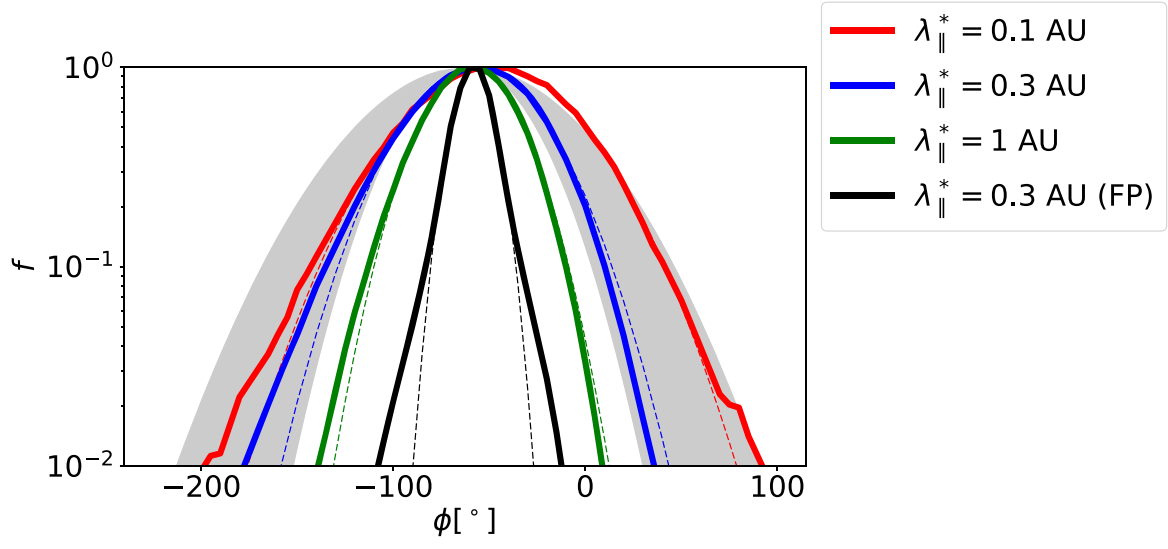

Fig. 6. The $10 \mathrm{MeV}$ SEP maximum intensity at $1 \mathrm{AU}$ during the first $10 \mathrm{~h}$ of the event, as a function of longitude. The thin dashed curves show the fitted Gaussian profiles with $\sigma_{\phi, \max }=41^{\circ}, 33^{\circ}, 23^{\circ}$ for $\lambda_{\|}^{*}=0.1 \mathrm{AU}$ (red curve), $0.3 \mathrm{AU}$ (blue curve) and $1 \mathrm{AU}$ (green curve), respectively, and $10^{\circ}$ for the reference FP simulation case with $\lambda_{\|}^{*}=0.3 \mathrm{AU}$ (black curve). The gray area depicts the observational range $\sigma_{\varphi, \text { max }}=30^{\circ}-50^{\circ}$.

focusing adiabatically outwards when they first arrive to 1 AU. The longitudinal width of the particle distribution is almost completely determined by the diffusive spreading of the field lines: there is no appreciable additional longitudinal widening of the particle distribution after the first two hours during the simulation period. This is most likely due to a combination of two effects: The cross-field particle diffusion coefficient for the case $\lambda_{\|}^{*}=1 \mathrm{AU}$ is half of that of the case $\lambda_{\|}^{*}=0.1 \mathrm{AU}$, thus less cross-field spreading of the particles can be expected. In addition, the particles escape from the inner heliosphere very efficiently due to adiabatic focusing and weak parallel scattering. Thus, the widening of the SEP distribution due to cross-field propagation of SEPs is compensated by the escape of particles to the outer heliosphere, resulting in almost constant intensity at longitudes far from the longitude $\varphi=-62^{\circ}$ connected to the SEP source along the Parker spiral.

The longitudinal extent of the SEP events, as observed using multiple spacecraft observations, is typically quantified by fitting a Gaussian curve to the observed peak intensities at different longitudes. Several case and statistical studies report the standard deviation $\sigma_{\varphi \text {, max }}$ of the Gaussian to be in the range of $30^{\circ}-50^{\circ}$ for both electrons and ions at different energies, in both gradual (Dresing et al., 2012; Lario et al., 2013; Richardson et al., 2014; Dresing et al., 2014) and impulsive SEP events (Wiedenbeck et al., 2013; Cohen et al., 2014). We present the longitudinal distribution of the peak intensities during the first 10 hours for our simulation cases in Figure 6, including the observational range $\sigma_{\varphi, \max }=30^{\circ}-50^{\circ}$ shown with the gray area, and the conventional Fokker-Planck result for $\lambda_{\|}^{*}=0.3 \mathrm{AU}$ with the black curve.

As can be seen, the longitudinal width of the SEP event at $1 \mathrm{AU}$ depends on the turbulence strength, with strong turbulence resulting in considerably wider SEP events, with $\sigma_{\varphi, \max }=41^{\circ}$ for the $\lambda_{\|}^{*}=0.1 \mathrm{AU}$ case, as compared to the narrow $\sigma_{\varphi, \max }=23^{\circ}$ for the $\lambda_{\|}^{*}=1 \mathrm{AU}$ case. We demonstrate the dependence of $\sigma_{\varphi \text {,max }}$ on the turbulence amplitude, as parametrised by $\lambda_{\|}^{*}$, in Figure 7 . The blue curve shows the expected trend for SEPs propagating solely along meandering field lines, which implies $\sigma_{\varphi, \text { max }}^{2} \sim D_{F L} \propto \delta B / B$ which, with 


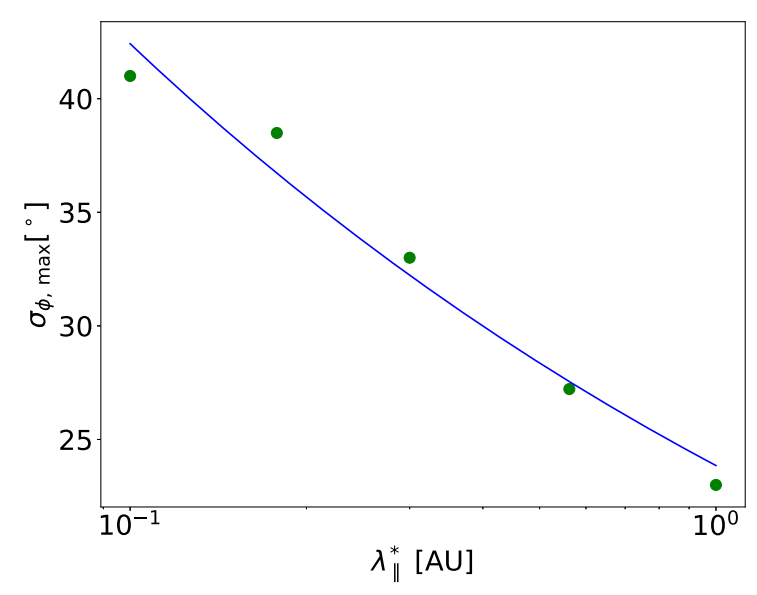

Fig. 7. The Gaussian $\sigma_{\varphi \text {,max }}$ fitted to the $10 \mathrm{MeV}$ SEP maximum intensity at $1 \mathrm{AU}$ during the first $10 \mathrm{~h}$ of the event, as a function of mean free path (green circles). The blue curve depicts the trend $\sigma_{\phi, \max } \propto\left(1 / \lambda_{\|}^{*}\right)^{1 / 4}$ expected for particles propagating along meandering field lines.

$\lambda_{\|} \propto B^{2} / \delta B^{2}$ results in $\sigma_{\varphi, \max } \propto\left(1 / \lambda_{\|}^{*}\right)^{1 / 4}$. As shown in Figure 7, our model results follow this scaling well. The slight deviation of the expected $\sigma_{\varphi, \max } \alpha\left(1 / \lambda_{\|}^{*}\right)^{1 / 4}$ trend is likely to be caused by more efficient cross-field diffusion of particles from the meandering field lines by the peak time for small $\lambda_{\|}^{*}$, as the peak times will be progressively later for smaller $\lambda_{\| \cdot}^{*}$

Also evident in the longitudinal distribution of the SEPs in Figure 6 is the asymmetry of the distribution with respect to the longitude connected to the injection site, $\varphi=-62^{\circ}$. The centres of the Gaussians are shifted to the West, with the longitude of the centre of the Gaussian $\varphi_{\max }=-48^{\circ}$ for the strong turbulence case with $\lambda_{\|}^{*}=0.1 \mathrm{AU}$. For weaker turbulence cases, $\varphi_{\max }$ approaches the best-connected longitude, with $-57^{\circ}$ and $-59^{\circ}$ for the $\lambda_{\|}^{*}=0.3 \mathrm{AU}$ and $1 \mathrm{AU}$ cases, respectively. Similar shifts were also found in simulations by Strauss and Fichtner (2015) and Strauss and Fichtner (2015). A shift of the maximum of around $10-15^{\circ}$ to the West has been reported in multispacecraft observed SEP events (Lario et al., 2006, 2013; Richardson et al., 2014). It should be noted though that the multi-spacecraft measurements are typically performed using a maximum of three measurement points, which makes estimation of the exact shape and asymmetries of the longitudinal distributions difficult. In addition, Richardson et al. (2014) reported the centre of the longitudinal peak distribution as $15^{\circ} \pm 35^{\circ}$ west of the connected longitude, emphasising the large errors associated in both determining the SEP source location and errors in inferring the magnetic connection of the observing spacecraft to the source location.

\section{Discussion}

Our study shows that meandering field lines are able to efficiently spread SEPs across the mean Parker Spiral direction at wide range of heliospheric turbulence conditions, even in weak scattering conditions. The propagation of SEPs along meandering field lines results in longitudinally wide SEP events, with dependence of the longitudinal width scaling with the SEP parallel mean free path as $\sigma_{\phi, \max } \propto\left(1 / \lambda_{\|}^{*}\right)^{1 / 4}$. The evolution of the SEP event after the initial phase is strongly dependent on the amount of turbulence in the heliosphere. In strong scattering environment, the longitudinal extent of the SEPs increases diffusively, (Fig. 4), whereas in the weak turbulence case, after the initial fast expansion, the longitudinal extent of the SEP event remains unchanged for the first $10 \mathrm{~h}$ (Fig. 5).

Our results also emphasise the importance of correctly accounting for the link between the interplanetary turbulence conditions and the particle transport coefficients. High turbulence amplitudes result in strong particle scattering along the mean field direction, and hence a short parallel mean free path (e.g. Jokipii, 1966) The particle propagation across the mean field, on the other hand is more efficient in stronger turbulence, as shown in both simulation studies (e.g. Giacalone and Jokipii, 1999; Laitinen and Dalla, 2017; Laitinen et al., 2017 and theoretical work (e.g. Matthaeus et al., 2003; Shalchi, 2010; Ruffolo et al., 2012). This can be clearly seen in Figure 1, where the evolution of the parallel and perpendicular scattering mean free paths are anticorrelated. Using quasilinear theory and the field-line diffusion coefficient from Matthaeus et al. (1995), $D_{F L}$, we find $D_{F L} \propto 1 / \sqrt{\lambda_{\|}}$, a scaling which is also consistent with Dröge et al. (2016) (their Fig. 17). Also, changing the geometry of the turbulence from the often-used $\delta B_{\|}^{2} / \delta B_{\perp}^{2}$ energy ratio $20 \%: 80 \%$ would have an influence on the SEP event evolution: increasing the proportion of the $2 \mathrm{D}$ component would result in faster onsets with wider cross-field extents. The overall dependence of $\varphi_{\max }\left(\lambda_{\|}^{*}\right)$ shown in Figure 7 would however, likely stay similar for a fixed $\delta B_{\|}^{2} / \delta B_{\perp}^{2}$ ratio. Other refinements of turbulence modelling, such as incorporating scale-dependence for the $k_{\perp} / k_{\|}$anisotropy (Goldreich and Sridhar, 1995; Shalchi et al., 2010; Laitinen et al., 2013a) and dynamical evolution of the turbulence (accounted for parallel propagation in Bieber et al., 1994), would naturally affect both early and late cross-field evolution of particle populations. These are left for future studies.

The interdependency between the parallel and cross-field SEP transport parameters, is typically ignored in parametric 3D SEP transport studies (e.g. Zhang et al., 2009; Dröge et al., 2010; He et al., 2011; Giacalone and Jokipii, 2012), and ad-hoc values are typically used. While the recent studies by Laitinen et al. (2016) and Strauss et al. (2017) did model SEP propagation with consistently modelled SEP transport coefficients or one set of turbulence parameters, our paper is to our knowledge the first to consistently study the effect of varying turbulence strength on both parallel and cross-field propagation when modelling SEP events in 3D.

The turbulence parameters are typically observed using insitu instruments onboard individual spacecraft, providing a single-point measurement of the turbulence properties. The SEPs, however, propagate across the mean field, sampling different heliolatitudes and longitudes, and their propagation is affected by the 3D turbulent structure of the heliosphere. Thus, to improve our ability to estimate the radiation environment in nearEarth space, we should consider a 3D picture of turbulence in the heliosphere. The need for longitudinally resolved particle transport conditions was recently highlighted also by Dröge et al. (2016), who found that the SEP intensities observed by the STEREO and ACE spacecraft during a single SEP event may require different diffusion coefficients for fitting the SEP observations at different longitudes. Recent work by Thomas et al. (2017) has shown promise of using solar wind observations at the Lagrangian point L5, $60^{\circ}$ behind Earth at Earth's orbit, for 
forecasting the solar wind conditions at L1. Such forecast would also make it possible to evaluate either the average or longitudinally dependent SEP transport parameters at a wide range of longitudes, from L5 to Earth and beyond. The recently proposed space weather missions to L5 (Akioka et al., 2005; Gopalswamy et al., 2011; Lavraud et al., 2016; Trichas et al., 2015 ) thus could bring considerable improvement to our ability to model SEP events and improve our knowledge of the radiation environment in near-Earth space.

\section{Conclusions}

We have studied how the strength of the turbulence in the interplanetary medium affects SEP event evolution within the new paradigm introduced by L2013 that includes the early non-diffusive cross-field transport of SEPs along meandering field lines. We found that

- the parallel and cross-field transport of SEPs are inherently linked through the turbulence properties, with high levels of turbulence resulting in diffusively spreading wide, gradually-rising SEP events, and low turbulence in fast SEP events which remain at nearly constant longitudinal extent after the initial rapid cross-field spreading along meandering field lines;

- the longitudinal distribution of $10 \mathrm{MeV}$ proton peak intensity follows approximately a Gaussian shape, with the longitudinal width of the distribution scaling as $\left(1 / \lambda_{\|}^{*}\right)^{1 / 4}$;

- in strong turbulence, the longitudinal distribution of the particles is asymmetric with respect to the longitude connected to the injection site, with the center of the fitted Gaussian distribution shifted by $14^{\circ}$ to the west. Weaker turbulence cases are less skewed with respect to the connected longitude.

Our results show that knowledge of the turbulence conditions of the heliospheric plasmas is crucial for modelling the cross-field propagation of the SEPs early in the events. To forecast the particle radiation conditions at Earth due to solar eruptions we must understand the full chain of phenomena including the injection of the particles at Sun, the physics behind their propagation in the interplanetary medium, and state of the interplanetary turbulence during the SEP propagation within the heliosphere.

Acknowledgements. TL and SD acknowledge support from the UK Science and Technology Facilities Council (STFC) (grants $\mathrm{ST} / \mathrm{J} 001341 / 1$ and ST/M00760X/1), and FE from NASA grants NNX14AG03G and NNX17AK25G. The contribution of AK benefited from financial support through project $\mathrm{He}$ 3279/15-1, funded by the Deutsche Forschungsgemeinschaft (DFG), at the CAU Kiel, where large parts of this work were carried out. Access to the University of Central Lancashire's High Performance Computing Facility is gratefully acknowledged. The editor thanks R. Du Toit Strauss and an anonymous referee for their assistance in evaluating this paper.

\section{References}

Akioka M, Nagatsuma T, Miyake W, Ohtaka K, Marubashi K. 2005. The L5 mission for space weather forecasting. Adv Space Res 35 : 65-69. DOI:10.1016/j.asr.2004.09.014.
Bavassano B, Dobrowolny M, Mariani F, Ness NF. 1982. Radial evolution of power spectra of interplanetary Alfvenic turbulence. $J$ Geophys Res 87: 3617-3622. DOI:10.1029/JA087iA05p03617.

Beeck J, Wibberenz G. 1986. Pitch angle distributions of solar energetic particles and the local scattering properties of the interplanetary medium. ApJ 311: 437-450. DOI: 10.1086/ 164784.

Bieber JW, Matthaeus WH, Smith CW, Wanner W, Kallenrode M-B, Wibberenz G. 1994. Proton and electron mean free paths: the palmer consensus revisited. ApJ 420: 294-306. DOI:10.1086/ 173559.

Bieber JW, Wanner W, Matthaeus WH. 1996. Dominant twodimensional solar wind turbulence with implications for cosmic ray transport. J Geophys Res 101: 2511-2522. DOI:10.1029/ 95JA02588.

Bruno R, Carbone V. 2013. The solar wind as a turbulence laboratory. Living Rev Sol Phys 10: 2. DOI:10.12942/lrsp-2013-2.

Burlaga LF, Turner JM. 1976. Microscale 'Alfven waves' in the solar wind at 1 AU. J Geophys Res 81: 73-77. DOI:10.1029/ JA081i001p00073.

Burger RA, Potgieter MS, Heber B. 2000. Rigidity dependence of cosmic ray proton latitudinal gradients measured by the Ulysses spacecraft: implications for the diffusion tensor. J Geophys Res 105: 447-456. DOI:10.1029/2000JA000153.

Chhiber R, Subedi P, Usmanov AV, Matthaeus WH, Ruffolo D, Goldstein ML, Parashar TN. 2017. Cosmic ray diffusion coefficients throughout the inner heliosphere from global solar wind simulation. ArXiv e-prints.

Cohen CMS, Mason GM, Mewaldt RA, Wiedenbeck ME. 2014. The longitudinal dependence of heavy-ion composition in the 2013 april 11 solar energetic particle event. ApJ 793: 35. DOI:10.1088/ 0004-637X/793/1/35.

Committee on the Evaluation of Radiation Shielding for Space Exploration, N. R. C, Managing Space Radiation Risk in the New Era of Space Exploration, The National Academies Press, Washington, DC, 2008. ISBN 978-0-309-11383-0.

Dalla S, Marsh MS, Laitinen T. 2015. Drift-induced deceleration of solar energetic particles. ApJ 808: 62. DOI:10.1088/0004-637X/ 808/1/62.

Dresing N, Gómez-Herrero R, Klassen A, Heber B, Kartavykh Y, Dröge W. 2012. The large longitudinal spread of solar energetic particles during the 17 january 2010 solar event. Sol Phys 281: 281-300. DOI:10.1007/s11207-012-0049-y.

Dresing N, Gómez-Herrero R, Heber B, Klassen A, Malandraki O, Dröge W, Kartavykh Y. 2014. Statistical survey of widely spread out solar electron events observed with STEREO and ACE with special attention to anisotropies. $A \& A$ 567: A27. DOI:10.1051/ 0004-6361/201423789.

Dröge W, Kartavykh YY, Klecker B, Kovaltsov GA. 2010. Anisotropic three-dimensional focused transport of solar energetic particles in the inner heliosphere. ApJ 709: 912-919. DOI:10.1088/ 0004-637X/709/2/912.

Dröge W, Kartavykh YY, Dresing N, Heber B, Klassen A. 2014. Wide longitudinal distribution of interplanetary electrons following the 7 February 2010 solar event: observations and transport modeling. $J$ Geophys Res: Space Phys 119: 6074-6094. DOI:10.1002/ 2014JA019933.

Dröge W, Kartavykh YY, Dresing N, Klassen A. 2016. Multispacecraft observations and transport modeling of energetic electrons for a series of solar particle events in august 2010. ApJ 826: 134. DOI: $10.3847 / 0004-637 \mathrm{X} / 826 / 2 / 134$.

Gardiner CW, Stochastic methods, vol. 13, 4th edn., Springer-Verlag, Berlin Heidelberg, 2009. 
Giacalone J. 2001. The latitudinal transport of energetic particles associated with corotating interaction regions. J Geophys Res 106: 15881-15888. DOI:10.1029/2000JA000114.

Giacalone J, Jokipii JR. 1999. The transport of cosmic rays across a turbulent magnetic field. ApJ 520: 204-214. DOI:10.1086/307452.

Giacalone J, Jokipii JR. 2012. The longitudinal transport of energetic ions from impulsive solar flares in interplanetary space. ApJL 751: L33. DOI:10.1088/2041-8205/751/2/L33.

Goldreich P, Sridhar S. 1995. Toward a theory of interstellar turbulence. 2: Strong alfvenic turbulence. ApJ 438: 763-775. DOI:10.1086/175121.

Gopalswamy N, Davila JM, St. Cyr OC, Sittler EC, Auchère F, et al. 2011. Earth-affecting solar causes observatory (easco): a potential inte rnational living with a star mission from sun-earth L5. J Atmos Sol-Terr Phys 73: 658-663. DOI:10.1016/j.jastp.2011.01.013.

Gray PC, Pontius DH, Matthaeus WH. 1996. Scaling of field-line random walk in model solar wind fluctuations. Geophys Res Lett 23: 965-968. DOI:10.1029/96GL00769.

He H-Q, Qin G, Zhang M. 2011. Propagation of solar energetic particles in three-dimensional interplanetary magnetic fields: in view of characteristics of sources. ApJ 734: 74. DOI:10.1088/ 0004-637X/734/2/74.

Isenberg PA. 1997. A hemispherical model of anisotropic interstellar pickup ions. J Geophys Res 102: 4719-4724. DOI:10.1029/ 96JA03671.

Jokipii JR. 1966. Cosmic-ray propagation. I. Charged particles in a random magnetic field. ApJ 146: 480-487. DOI:10.1086/148912.

Kopp A, Büsching I, Strauss RD, Potgieter MS. 2012. A stochastic differential equation code for multidimensional Fokker-Planck type problems. Comput Phys Commun 183: 530-542. DOI:10.1016/j.cpc.2011.11.014.

Kóta J, Jokipii JR. 2000. Velocity correlation and the spatial diffusion coefficients of cosmic rays: compound diffusion. ApJ 531: 10671070. DOI:10.1086/308492.

Laitinen T, Dalla S. 2017. Energetic particle transport across the mean magnetic field: before diffusion. ApJ 834: 127. DOI:10.3847/ 1538-4357/834/2/127.

Laitinen T, Dalla S, Kelly J, Marsh M. 2013a. Energetic particle diffusion in critically balanced turbulence. ApJ 764: 168. DOI:10.1088/0004-637X/764/2/168.

Laitinen T, Dalla S, Marsh MS. 2013b. Energetic particle cross-field propagation early in a solar event. ApJL 773: L29. DOI:10.1088/ 2041-8205/773/2/L29.

Laitinen T, Kopp A, Effenberger F, Dalla S, Marsh MS. 2016. Solar energetic particle access to distant longitudes through turbulent field-line meandering. $A \& A$ 591: A18. DOI:10.1051/0004-6361/ 201527801.

Laitinen T, Dalla S, Marriott D. 2017. Early propagation of energetic particles across the mean field in turbulent plasmas. Mon Not $R$ Astron Soc 470: 3149-3158. DOI:10.1093/mnras/stx1509.

Lario D, Kallenrode M-B, Decker RB, Roelof EC, Krimigis SM, Aran A, Sanahuja B. 2006. Radial and longitudinal dependence of solar 4-13 MeV and 27-37 MeV proton peak intensities and fluences: helios and IMP 8 observations. ApJ 653: 1531-1544. DOI:10.1086/ 508982.

Lario D, Aran A, Gómez-Herrero R, Dresing N, Heber B, Ho GC, Decker RB, Roelof EC. 2013. Longitudinal and radial dependence of solar energetic particle peak intensities: STEREO, ACE, SOHO, GOES, and MESSENGER Observations. ApJ 767: 41. DOI:10.1088/0004-637X/767/1/41.

Lavraud B, Liu Y, Segura K, He J, Qin G, et al. 2016. A small mission concept to the SunEarth Lagrangian $\{$ L5 $\}$ point for innovative solar, heliospheric and space weather science. J Atmos Sol-Terr
Phys 146: 171-185. DOI:10.1016/j.jastp.2016.06.004. //www. sciencedirect.com/science/article/pii/S1364682616301456.

Matthaeus WH, Gray PC, Pontius DH Jr., Bieber JW. 1995. Spatial structure and field-line diffusion in transverse magnetic turbulence. Phys Rev Lett 75: 2136-2139. DOI:10.1103/PhysRevLett.75.2136.

Matthaeus WH, Qin G, Bieber JW, Zank GP. 2003. Nonlinear collisionless perpendicular diffusion of charged particles. ApJL 590: L53-L56. DOI:10.1086/376613.

Mazur JE, Mason GM, Dwyer JR, Giacalone J, Jokipii JR, Stone EC. 2000. Interplanetary magnetic field line mixing deduced from impulsive solar flare particles. ApJL 532: L79-L82. DOI:10.1086/ 312561.

Palmer ID. 1982. Transport coefficients of low-energy cosmic rays in interplanetary space. Rev Geophys Space Phy 20: 335-351.

Parker EN. 1965. The passage of energetic charged particles through interplanetary space. Planet Space Sci 13: 9-49. DOI:10.1016/ 0032-0633(65)90131-5.

Pei C, Bieber JW, Breech B, Burger RA, Clem J, Matthaeus WH. 2010. Cosmic ray diffusion tensor throughout the heliosphere. $J$ Geophys Res: Space Phys 115: A03103. DOI:10.1029/ 2009JA014705.

Potgieter MS, Vos EE, Boezio M, De Simone N, Di Felice V, Formato V. 2014. Modulation of galactic protons in the heliosphere during the unusual solar minimum of 2006 to 2009. Sol Phys 289: 391406. DOI:10.1007/s11207-013-0324-6.

Qin G, Shalchi A. 2014. Pitch-angle dependent perpendicular diffusion of energetic particles interacting with magnetic turbulence. Appl Phys Res 6: 1-13.

Reames DV. 1999. Particle acceleration at the Sun and in the heliosphere. Space Sci Rev 90: 413-491. DOI:10.1023/ A:1005105831781.

Richardson IG, von Rosenvinge TT, Cane HV, Christian ER, Cohen CMS, Labrador AW, Leske RA, Mewaldt RA, Wiedenbeck ME, Stone EC. 2014. $25 \mathrm{MeV}$ Proton events observed by the high energy telescopes on the STEREO A and B spacecraft and/or at earth during the first $\sim$ seven years of the STEREO mission. Sol Phys 289: 3059-3107. DOI:10.1007/s11207-014-0524-8.

Richter AK, Olbers DJ. 1974. Wave-trains in the solar wind. II: comments on the propagation of alfvén waves in the quiet interplanetary medium. Astrophys Space Sci 26: 95-105. DOI:10.1007/BF00642623.

Roelof EC. 1969. Propagation of solar cosmic rays in the interplanetary magnetic field. In H. Ögelman, JR. Wayland, eds. Lectures in High-Energy Astrophysics. 111 p.

Ruffolo D, Matthaeus WH, Chuychai P. 2004. Separation of magnetic field lines in two-component turbulence. ApJ 614: 420-434. DOI: $10.1086 / 423412$

Ruffolo D, Pianpanit T, Matthaeus WH, Chuychai P. 2012. Random ballistic interpretation of nonlinear guiding center theory. ApJL 747: L34. DOI:10.1088/2041-8205/747/2/L34.

Shalchi A. 2010. A unified particle diffusion theory for cross-field scattering: subdiffusion, recovery of diffusion, and diffusion in three-dimensional turbulence. ApJL 720: L127-L130. DOI: $10.1088 / 2041-8205 / 720 / 2 /$ L127.

Shalchi A, Büsching I, Lazarian A, Schlickeiser R. 2010. Perpendicular diffusion of cosmic rays for a goldreich-sridhar spectrum. ApJ 725: 2117-2127. DOI:10.1088/0004-637X/725/2/ 2117.

Skilling J. 1971. Cosmic rays in the galaxy: convection or diffusion? ApJ 170: 265. DOI:10.1086/151210.

Strauss RD, Fichtner H. 2015. On aspects pertaining to the perpendicular diffusion of solar energetic particles. ApJ 801: 29, 10.1088/0004-637X/801/1/29. 
Strauss RDT, Effenberger F. 2017. A hitch-hiker's guide to stochastic differential equations. Space Sci Rev 1-42. DOI:10.1007/ s11214-017-0351-y. http://dx.doi.org/10.1007/s11214-017-0351-y

Strauss RDT, Dresing N, Engelbrecht NE. 2017. Perpendicular diffusion of solar energetic particles: model results and implications for electrons. ApJ 837: 43. DOI:10.3847/1538-4357/ aa5df5.

Thomas SR, Fazakerley AN, Wicks RT, Green L. 2017. Evaluating the skill of forecasts of the near-earth solar wind using a space weather monitor at L5. Space Weather J, submitted.

Tooprakai P, Seripienlert A, Ruffolo D, Chuychai P, Matthaeus WH. 2016. Simulations of lateral transport and dropout structure of energetic particles from impulsive solar flares. ApJ 831: 195. DOI:10.3847/0004-637X/831/2/195.

Trichas M, Gibbs M, Harrison R, Green L, Eastwood J, Bentley B, et al. 2015. Carrington-L5: the UK/US operational space weather monitoring mission. Hipparchos 2: 25-31.
Tu C-Y, Pu Z-Y, Wei F-S. 1984. The power spectrum of interplanetary alfvenic fluctuations derivation of the governing equation and its solution. J Geophys Res 89: 9695-9702. DOI:10.1029/JA089i A11p09695.

Wanner W, Wibberenz G. 1993. A study of the propagation of solar energetic protons in the inner heliosphere. J Geophys Res 98: 3513 3528. DOI:10.1029/92JA02546.

Wiedenbeck ME, Mason GM, Cohen CMS, Nitta NV, GómezHerrero R, Haggerty DK. 2013. Observations of solar energetic particles from ${ }^{3} \mathrm{He}$-rich Events over a wide range of heliographic longitude. ApJ 762: 54. DOI:10.1088/0004-637X/762/1/54.

Zhang M, Jokipii JR, McKibben RB. 2003. Perpendicular transport of solar energetic particles in heliospheric magnetic fields. ApJ 595: 493-499. DOI:10.1086/377301.

Zhang M, Qin G, Rassoul H. 2009. Propagation of solar energetic particles in three-dimensional interplanetary magnetic fields. $A p J$ 692: 109-132. DOI:10.1088/0004-637X/692/1/109.

Cite this article as: Laitinen T, Effenberger F, Kopp A, Dalla S. 2018. The effect of turbulence strength on meandering field lines and Solar Energetic Particle event extents. J. Space Weather Space Clim. 8: A13 